\title{
Advocating neuroimaging studies of transmitter release in human physical exercise challenges studies
}

This article was published in the following Dove Press journal:

Open Access Journal of Sports Medicine

4 September 2010

Number of times this article has been viewed

\author{
Henning Boecker \\ Ahmed Othman' \\ Sarah Mueckter' \\ Lukas Scheef' \\ Max Pensel' \\ Marcel Daamen' \\ Jakob Jankowski' \\ $\mathrm{HH}$ Schild ${ }^{2}$ \\ TR Tölle ${ }^{3}$ \\ M Schreckenberger ${ }^{4}$ \\ 'FE Klinische Funktionelle \\ Neurobildgebung, Radiologische \\ Universitätsklinik, Friedrich- \\ Wilhelms-Universität Bonn, Germany; \\ ${ }^{2}$ Radiologische Universitätsklinik, \\ Friedrich-Wilhelms-Universität Bonn, \\ Germany; ${ }^{3}$ TUM Neurologische \\ Klinik und Poliklinik im Neuro- \\ Kopf-Zentrum, Klinikum rechts der \\ Isar der Technischen Universität \\ München, München, Germany; ${ }^{4} \mathrm{Klinik}$ \\ und Poliklinik für Nuklearmedizin \\ am Mainzer Universitätsklinikum, \\ Johannes Gutenberg-Universität, \\ Mainz, Germany
}

Correspondence: Henning Boecker Head FE Klinische Funktionelle Neurobildgebung, Radiologische Universitätsklinik, Rheinische FriedrichWilhelms-Universität Bonn, SigmundFreud-Str. 25, 53 27 Bonn, Germany Tel +49 (0)228 287 I5970/80 Fax +49 (0)228 287 I4457 Email henning.boecker@ukb.uni-bonn.de
Abstract: This perspective attempts to outline the emerging role of positron emission tomography (PET) ligand activation studies in human exercise research. By focusing on the endorphinergic system and its acclaimed role for exercise-induced antinociception and mood enhancement, we like to emphasize the unique potential of ligand PET applied to human athletes for uncovering the neurochemistry of exercise-induced psychophysiological phenomena. Compared with conventional approaches, in particular quantification of plasma beta-endorphin levels under exercise challenges, which are reviewed in this article, studying opioidergic effects directly in the central nervous system (CNS) with PET and relating opioidergic binding changes to neuropsychological assessments, provides a more refined and promising experimental strategy. Although a vast literature dating back to the 1980 s of the last century has been able to reproducibly demonstrate peripheral increases of beta-endorphin levels after various exercise challenges, so far, these studies have failed to establish robust links between peripheral beta-endorphin levels and centrally mediated behavioral effects, ie, modulation of mood and/or pain perception. As the quantitative relation between endorphins in the peripheral blood and the CNS remains unknown, the question arises, to what extent conventional blood-based methods can inform researchers about central neurotransmitter effects. As previous studies using receptor blocking approaches have also revealed equivocal results regarding exercise effects on pain and mood processing, it is expected that PET and other functional neuroimaging applications in athletes may in future help uncover some of the hitherto unknown links between neurotransmission and psychophysiological effects related to physical exercise.

Keywords: positron emission tomography, beta-endorphins, opioids

\section{Introduction}

Regular physical exercise is associated with a wide spectrum of psychophysical effects, including anxiolysis, ${ }^{1}$ stress reduction, ${ }^{2}$ mood elevation, ${ }^{1,3-5}$ and altered pain perception. ${ }^{3}$ The underlying neurotransmitter effects (eg, dopaminergic, opioidergic, endocannabinoid, and serotonergic) in the central nervous system (CNS) and their specific roles for induction and maintenance of distinct psychophysiological phenomena are subject to both nonhuman and human exercise research. Although animal studies allow direct quantitative and regional assessments of neurotransmitter trafficking in the CNS via invasive microdialysis measurements in exercising animals, ${ }^{6}$ or postmortem autoradiography of receptor binding changes after exercise, ${ }^{7}$ until recently, human studies examining neuro-humeral effects of exercise have been derived exclusively from indirect peripheral neurotransmitter levels in plasma and receptor blockade studies (discussed later). 
The central endorphinergic system, which is the focus of this article, has been linked to exercise-induced mood changes ${ }^{1,3,4,8-11}$ and antinociceptive effects referred to as stress-induced analgesia. ${ }^{3,9,10}$ The endorphinergic system can be studied in human athletes via measurements of peripheral beta-endorphin material, receptor blocking studies, or, more directly using positron emission tomography (PET) ligand displacement approaches (discussed later). This perspective, by contrasting the data derived from peripheral beta-endorphin measurements with initial PET studies in athletes, aims at highlighting the unique and unprecedented potential of PET ligand activation studies for exploring central neurotransmission related to physical exercise.

\section{Measurements of peripheral beta-endorphin levels in exercise studies}

Endogenous opioid peptides (endorphins, enkephalins, and dynorphins) interact with $\mu, \kappa$, and $\delta$ opioid receptors located in the CNS and the peripheral nervous system. During vigorous exercise, beta-endorphins are released from the pituitary gland into the blood, although with considerable intraindividual variability ${ }^{12}$ and inter-individual variability. ${ }^{13,14}$ Table 1 summarizes published data on this topic, as compiled from a PubMed search: "Exercise and Human and Plasma and Endorphin', which revealed 185 hits spanning from 1982 to 2008. We excluded work examining the effects of resistance training on beta-endorphin plasma levels, ${ }^{15}$ and we also excluded studies conducted in patient populations. Our inquiry resulted in 65 studies covering a wide range of physical exercise challenges from low to maximal intensity. Although we can make no claim of completeness regarding included studies, the summarized papers indicate that a high percentage of exercise-induced beta-endorphin plasma elevations. As can be seen from Table 1, 59 of 65 papers identified significant increases of peripheral endorphin values, despite highly heterogeneous exercise challenges. Studies applying different exercise intensity levels have shown a positive relationship between the intensity of exercise challenges and the magnitude of peripheral endorphin increases in plasma. ${ }^{16-27}$

\section{Link between peripheral beta-endorphin levels and mood and pain assessments}

Despite these highly reproducible increases of beta-endorphins in peripheral blood after exercise, as shown in Table 1, the correlation of peripheral beta-endorphin values with behavioral measures of altered mood or pain processing has yielded equivocal results. ${ }^{28}$ This may be linked to the fact that most of these large molecules can only bypass the blood-brain barrier to a very minor extent. ${ }^{29}$ Table 2 summarizes those studies that have correlated peripheral endorphin values after exercise challenges with changes in mood states, whereas Table 3 summarizes those studies that have correlated peripheral endorphin values after exercise challenges with pain scores. It can be seen from Table 2 that the association between peripheral beta-endorphin values and mood is indeed highly inconsistent, with only two out of 7 studies showing a positive relationship between both factors. ${ }^{3,30}$ Only 3 studies, we are aware of, have tested the relationship between exercise-induced peripheral beta-endorphin values and changes in pain ratings (Table 3). All of them have demonstrated exercise-induced hypoalgesia; however, only two of these identified a positive relationship between endorphins and hypoalgesia., ${ }^{3,31}$

Based on the available limited evidence, at present no clear relationship between peripheral endorphin levels and modulation of mood/pain processing can be established, thus arguing against a linear relationship between the peripheral and the central opioidergic compartments. Moreover, these negative findings are also at odds with the acclaimed role of exercise in promoting antinociception and mood enhancement. Therefore, we conclude that peripheral measurements of endorphins provide only limited information about central opioidergic mechanisms underlying psychophysiological effects. This also seems to apply to receptor blocking studies, which are not summarized here in detail, but which have also revealed equivocal results, ie, either negative $e^{32-35}$ or positive blocking effects in the pain domain. ${ }^{3,36}$ On the other hand, several studies have reported positive blocking effects in the mood domain, ${ }^{3,37,38}$ thus supporting the hypothesis of central opioidergic effects mediating mood enhancement. Alternatively, quantification of neurotransmitter levels in the cerebrospinal fluid compartment of the $\mathrm{CNS}^{39}$ seems to provide a more direct approach, and may yield more distinct information than plasma values. Yet, given the invasiveness of repetitive spinal fluid taps, this experimental approach has to be refuted for ethical reasons in humans. Moreover, any quantitative analysis of neurotransmitters in spinal fluid or plasma will not inform researchers about the site of neurotransmitter actions in the CNS and, therefore, will not be able to establish precise correlations with neurobehavioral measures.

\section{PET ligand activation of the opioidergic system}

PET studies allow noninvasively quantifying receptor binding of PET ligands within the entire CNS and, more recently, 
Table I Papers reporting peripheral beta-endorphin values in exercise challenges

\begin{tabular}{|c|c|c|c|c|c|c|c|}
\hline Publication & N/sex & Age & $\begin{array}{l}\text { Fitness } \\
\text { state }\end{array}$ & $\begin{array}{l}\text { Exercise } \\
\text { type }\end{array}$ & $\begin{array}{l}\text { Duration/ } \\
\text { distance }\end{array}$ & $\begin{array}{l}\text { Intensity } \\
\text { level }\end{array}$ & $\begin{array}{l}\text { Significance } \\
\text { (endorphine } \\
\text { increase) }\end{array}$ \\
\hline 48 & $9 / M$ & $27.6 \pm 1.6$ & Highly fit & Treadmill & $30 \mathrm{~min}$ & $80 \% \mathrm{VO}_{2} \max$ & $P<0.05$ \\
\hline 49 & $15 / M$ & $28.5 \pm 9.5$ & Unfit & Cycle ergometer & $60 \mathrm{~min}$ & $\begin{array}{l}60 \% \mathrm{VO}_{2} \max \\
80 \% \mathrm{VO}_{2} \max \end{array}$ & Significant \\
\hline 50 & $10 / M$ & $32.3 \pm 10.6$ & Fit & Treadmill & $30 \mathrm{~s}$ & $424.8 \pm 41.9 \mathrm{~W}$ & $P<0.001$ \\
\hline 16 & $7 / F$ & $24.6 \pm 4.2$ & Fit & Cycle ergometer & $60 \mathrm{~min}$ & $\begin{array}{l}20 \min 50 \% \\
20 \min 70 \% \\
10 \min 80-85 \% \\
\mathrm{VO}_{2} \max \end{array}$ & $\begin{array}{l}\text { NS } \\
\text { NS } \\
P<0.05\end{array}$ \\
\hline 51 & $\begin{array}{l}24 / \mathrm{M} \\
16 / \mathrm{F}\end{array}$ & $\begin{array}{l}12.85 \pm 0.054 \\
10.87 \pm 0.47\end{array}$ & Fit & Cycle ergometer & $15 \mathrm{~min}$ & $90 \% \mathrm{VO}_{2} \max$ & $P<0.01$ \\
\hline 52 & $13 / M$ & $18.6 \pm 0.7$ & Highly fit & $\begin{array}{l}\text { Swimming } \\
\text { (100 m freestyle) }\end{array}$ & $61.47 \pm 1.98 \mathrm{~s}$ & Competition condition & $P<0.01$ \\
\hline 53 & $\begin{array}{l}20 / M \\
6 / F\end{array}$ & $\begin{array}{l}34.4(27-42) \\
31.2(28-4 I)\end{array}$ & Highly fit & Outdoor running & $30 \mathrm{~min}$ & $\begin{array}{l}\text { "Easy run" } \\
\text { Maximal pace }\end{array}$ & $\begin{array}{l}P<0.067(\mathrm{NS}) \\
P<0.008\end{array}$ \\
\hline 54 & $\begin{array}{l}I I / M \\
8 / M\end{array}$ & $20-24$ & $\begin{array}{l}\text { Fit to highly fit } \\
\text { Unfit }\end{array}$ & Treadmill & - & $\begin{array}{l}\text { Graded intensity to } \\
\text { exhaustion }\end{array}$ & $P<0.05$ \\
\hline 55 & $\mathrm{II} / \mathrm{M}$ & - & - & Cycle ergometer & - & $\begin{array}{l}\text { Graded intensity to } \\
\text { exhaustion }\end{array}$ & $P<0.05$ \\
\hline 56 & I4/M & $26.7 \pm 3.2$ & Fit & Cycle ergometer & $60 \mathrm{~min}$ & $\begin{array}{l}112 \pm 16 \mathrm{~W} \\
(<70 \% \max H R)\end{array}$ & NS \\
\hline 17 & $8 / M$ & $21.9 \pm 2.4$ & - & Cycle ergometer & $40 \mathrm{~min}$ & $\begin{array}{l}40 \% \mathrm{VO}_{2} \max \\
60 \% \mathrm{VO}_{2} \max \\
80 \% \mathrm{VO}_{2} \max \\
100 \% \mathrm{VO}_{2} \max \\
10 \text { min each to } \\
\text { exhaustion }\end{array}$ & $\begin{array}{l}\text { NS } \\
\text { NS } \\
\text { NS } \\
P<0.01\end{array}$ \\
\hline 57 & $18 / M$ & $20.8 \pm 0.2$ & Highly fit & Treadmill & $30 \mathrm{~min}$ & Anaerobic threshold & NS \\
\hline 58 & $19 / M$ & $21.9 \pm 1.9$ & Unfit & Cycle ergometer & $32 \mathrm{~min}$ & $\begin{array}{l}\text { Graded intensity } \\
\text { to exhaustion }\end{array}$ & $P<0.05$ \\
\hline 59 & $10 / M$ & $33(20-46)$ & Fit & Cycle ergometer & - & $\begin{array}{l}\text { Graded intensity to } \\
\text { exhaustion }\end{array}$ & $P<0.000 I$ \\
\hline 60 & $8 / M$ & $26.8 \pm 8.6$ & Unfit & Treadmill & $20 \mathrm{~min}$ & $80 \% \max H R$ & NS \\
\hline 61 & $5 / M$ & - & Unfit & Treadmill & - & $\begin{array}{l}\text { Graded intensity } \\
\text { to exhaustion }\end{array}$ & $P<0.05$ \\
\hline 13 & $\begin{array}{l}5 / M \\
I / F\end{array}$ & $30.0 \pm 8.3$ & Highly fit & Treadmill & $30 \mathrm{~min}$ & $\begin{array}{l}60 \% \mathrm{VO}_{2} \max \\
80 \% \mathrm{VO}_{2} \max \end{array}$ & $\begin{array}{l}P<0.05 \\
\text { NS }\end{array}$ \\
\hline 18 & $\begin{array}{l}7 / M \\
7 / M\end{array}$ & $\begin{array}{l}23.1 \pm 2.5 \\
23.0 \pm 3.5\end{array}$ & $\begin{array}{l}\text { Fit } \\
\text { Unfit }\end{array}$ & Treadmill & $12 \mathrm{~min}$ & $\begin{array}{l}7 \text { min } 60 \% \\
\mathrm{VO}_{2} \max \\
3 \min 100 \% \\
\mathrm{VO}_{2} \max \\
2 \min 110 \% \mathrm{Vo}_{2} \max \end{array}$ & $\begin{array}{l}\text { NS } \\
P<0.05 \\
P<0.05\end{array}$ \\
\hline 62 & $10 / M$ & $26.3 \pm 5.4$ & Fit & Cycle ergometer & $120 \mathrm{~min}$ & $65 \% \mathrm{VO}_{2} \max$ & $P<0.05$ \\
\hline 63 & $2 \mathrm{I} / \mathrm{F}$ & 14 & Fit & Step test & $3-6 \min$ & To $66 \% \mathrm{VO}_{2} \max$ & $P<0.05$ \\
\hline 64 & $\begin{array}{l}8 / \mathrm{M} \\
7 / \mathrm{F}\end{array}$ & $\begin{array}{l}45.9 \pm 8.7 \\
46.3 \pm 5.3\end{array}$ & Highly fit & Outdoor running & $2 \mathrm{I}-42 \mathrm{~km}$ & Race conditions & $P<0.001$ \\
\hline 65 & $9 / M$ & $20-28$ & Unfit & Cycle ergometer & - & $\begin{array}{l}\text { Graded intensity } \\
\text { to exhaustion }\end{array}$ & $P<0.05$ \\
\hline 19 & $12 / M$ & $\begin{array}{l}26.5 \pm 1.3 \\
(21.37)\end{array}$ & - & Cycle ergometer & $30 \mathrm{~min}$ & $\begin{array}{l}60 \% \mathrm{Vo}_{2} \max \\
70 \% \mathrm{Vo}_{2} \max \\
80 \% \mathrm{Vo}_{2} \max \end{array}$ & $\begin{array}{l}\text { NS } \\
P<0.05 \\
P<0.05\end{array}$ \\
\hline 20 & $\begin{array}{l}6 / M \\
6 / M\end{array}$ & $\begin{array}{l}28.0 \pm 2.2 \\
25.0 \pm 1.4\end{array}$ & $\begin{array}{l}\text { Fit } \\
\text { Unfit }\end{array}$ & Cycle ergometer & $30 \mathrm{~min}$ & $\begin{array}{l}60 \% \mathrm{Vo}_{2} \max \\
70 \% \mathrm{Vo}_{2} \max \\
80 \% \mathrm{Vo}_{2} \max \end{array}$ & $\begin{array}{l}\text { NS } \\
P<0.05 \\
P<0.02\end{array}$ \\
\hline 21 & $12 / M+F$ & $26.4 / 26.8$ & - & Cycle ergometer & $2 \times 25 \min$ & $\begin{array}{l}60 \% \mathrm{VO}_{2} \max \\
80 \% \mathrm{VO}_{2} \max \end{array}$ & $\begin{array}{l}\text { NS } \\
P<0.05\end{array}$ \\
\hline
\end{tabular}


Table I (Continued)

\begin{tabular}{|c|c|c|c|c|c|c|c|}
\hline Publication & N/sex & Age & $\begin{array}{l}\text { Fitness } \\
\text { state }\end{array}$ & $\begin{array}{l}\text { Exercise } \\
\text { type }\end{array}$ & $\begin{array}{l}\text { Duration/ } \\
\text { distance }\end{array}$ & $\begin{array}{l}\text { Intensity } \\
\text { level }\end{array}$ & $\begin{array}{l}\text { Significance } \\
\text { (endorphine } \\
\text { increase) }\end{array}$ \\
\hline 66 & I4/M & $25.6 \pm 2.1$ & Fit & $\begin{array}{l}\text { Outdoor running }+ \\
\text { upstairs running } \\
\text { ( } 8 \text { floors) }\end{array}$ & $3 \mathrm{~km}$ & $\begin{array}{l}\text { Individual } \\
\text { maximal pace }\end{array}$ & $P<0.05$ \\
\hline 30 & $\mathrm{II} / \mathrm{M}$ & 31.3 & Highly fit & Outdoor running & $60 \mathrm{~min} / \mathrm{I} 5 \mathrm{~km}$ & $\begin{array}{l}\text { Fast training } \\
\text { pace }\end{array}$ & $P<0.0001$ \\
\hline 31 & $50 / F$ & - & - & Cycle ergometer & $20 \mathrm{~min}$ & $\begin{array}{l}\text { Moderate } \\
\text { intensity }\end{array}$ & $P<0.001$ \\
\hline 67 & $\begin{array}{l}9 / M \\
7 / M\end{array}$ & $\begin{array}{l}21.1 \pm 2.52 \\
66.0 \pm 5.85\end{array}$ & Unfit & Cycle ergometer & - & $\begin{array}{l}\text { Graded intensity } \\
\text { to exhaustion }\end{array}$ & $P<0.001$ \\
\hline 68 & $16 / M$ & 38 & Highly fit & $\begin{array}{l}\text { Cycle ergometer } \\
\text { Outdoor running }\end{array}$ & $-\overline{42 \mathrm{~km}}$ & $\begin{array}{l}\text { Graded intensity } \\
\text { to exhaustion } \\
\text { Race condition } \\
\left(83 \% \mathrm{VO}_{2} \max \right)\end{array}$ & $\begin{array}{l}P<0.001 \\
P<0.001\end{array}$ \\
\hline 69 & $14 / F$ & - & Highly fit & $\begin{array}{l}\text { Outdoor running } \\
\text { Treadmill running }\end{array}$ & $\begin{array}{l}3: 22 \text { h/42 km } \\
-\end{array}$ & $\begin{array}{l}\text { Race condition } \\
\text { To exhaustion }\end{array}$ & $\begin{array}{l}\text { Significant } \\
\text { Significant }\end{array}$ \\
\hline 70 & $23 / F$ & $21.7 \pm 1.9$ & Unfit & Treadmill & $30 \mathrm{~min}$ & $\begin{array}{l}\text { Graded intensity } \\
\text { to exhaustion }\end{array}$ & $P<0.05$ \\
\hline 71 & $5 / M$ & $22.6 \pm 1.3$ & Highly fit & Cycle ergometer & $60 \mathrm{~min}$ & $70 \% \mathrm{VO}_{2} \max$ & $P<0.05$ \\
\hline 72 & $I 8 / F$ & $20-23$ & Unfit & Treadmill & $60 \mathrm{~min}$ & $\begin{array}{l}60 \% \mathrm{VO}_{2} \max \\
70 \% \mathrm{Vo}_{2} \max \\
80 \% \mathrm{VO}_{2} \max \\
20 \text { min each }\end{array}$ & $P<0.01$ \\
\hline 3 & $I 2 / M$ & 38.3 & Highly fit & Outdoor running & $\begin{array}{l}43.9 \mathrm{~min} / \\
6.3 \mathrm{miles}\end{array}$ & $85 \% \mathrm{Vo}_{2} \max$ & $P<0.01$ \\
\hline 73 & $6 / M$ & $26.5 \pm 4.5$ & Fit & Cycle ergometer & $120 \mathrm{~min}$ & $50 \% \mathrm{VO}_{2} \max$ & $P<0.05$ \\
\hline 74 & I0/M & $23.9 \pm 3.8$ & - & Cycle ergometer & $<\mathrm{I}-4 \min$ & $\begin{array}{l}115 \% \mathrm{VO}_{2} \max \\
175 \% \mathrm{VO}_{2} \max \\
230 \% \mathrm{VO}_{2} \max \\
318 \% \mathrm{VO}_{2} \max \end{array}$ & $\begin{array}{l}P<0.05 \\
N S \\
N S \\
\text { NS }\end{array}$ \\
\hline 75 & $\begin{array}{l}8 / M+F \\
10 / M+F \\
7 / M+F\end{array}$ & $\begin{array}{l}26.5 \pm 5.0 \\
23.1 \pm 4.1 \\
21.9 \pm 3.1\end{array}$ & Unfit & Treadmill & - & $\begin{array}{l}\text { Maximal } \\
\text { intensity }\end{array}$ & $P<0.05$ \\
\hline 76 & $6 / M$ & $22 \pm 2$ & Fit & Cycle ergometer & $7-8 \mathrm{~min}$ & $\begin{array}{l}3 \min 90 \% \\
\mathrm{VO}_{2} \max \\
3-4 \min 100 \% \\
\mathrm{VO}_{2} \max \end{array}$ & $\begin{array}{l}P<0.05 \\
P<0.05\end{array}$ \\
\hline 77 & $\begin{array}{l}8 / \mathrm{M}, 5 / \mathrm{F} \\
5 / \mathrm{M}, 5 / \mathrm{F}\end{array}$ & $\begin{array}{l}31.5 \pm 30.4 \\
29.6 \pm 28.8\end{array}$ & $\begin{array}{l}\text { Fit } \\
\text { Unfit }\end{array}$ & Treadmill & $30 \mathrm{~min}$ & $80 \% \max H R$ & NS \\
\hline 78 & $\begin{array}{l}5 / M \\
5 / M\end{array}$ & $\begin{array}{l}22 \pm 2 \\
22 \pm 2\end{array}$ & $\begin{array}{l}\text { Fit } \\
\text { Fit }\end{array}$ & $\begin{array}{l}\text { Cycle ergometer } \\
\text { Treadmill }\end{array}$ & $\begin{array}{l}60 \mathrm{~min} \\
60 \mathrm{~min}\end{array}$ & $\begin{array}{l}60 \% \mathrm{VO}_{2} \max \\
0 \% \mathrm{VO}_{2} \max \end{array}$ & $\begin{array}{l}\text { NS } \\
\text { NS }\end{array}$ \\
\hline 22 & $20 / M$ & $26 \pm 1$ & Highly fit & Cycle ergometer & $\begin{array}{l}120 \min \\
120 \min + \\
I \text { min } \\
\text { sprint }\end{array}$ & $\begin{array}{l}120 \min 65 \% \\
\mathrm{VO}_{2} \max \\
120 \min 65 \% \\
\mathrm{VO}_{2} \max +\text { spints a } \\
120 \% \mathrm{VO}_{2} \max \end{array}$ & $\begin{array}{l}\text { NS } \\
P<0.001 \text { (after } \\
\text { sprints) }\end{array}$ \\
\hline 79 & $6 / M$ & $33.5 \pm 8.6$ & Fit & Cycle ergometer & - & $\begin{array}{l}\text { Graded intensity } \\
\text { to exhaustion }\end{array}$ & $P<0.05$ \\
\hline 23 & $\begin{array}{l}10 / \mathrm{M} \\
10 / \mathrm{F}\end{array}$ & $24.5 \pm 4.8$ & Unfit & Cycle ergometer & $3 \times 20 \min$ & $\begin{array}{l}40 \% \mathrm{VO}_{2} \max \\
60 \% \mathrm{VO}_{2} \max \\
80 \% \mathrm{VO}_{2} \max \\
20 \text { min each }\end{array}$ & $\begin{array}{l}\text { NS } \\
\text { NS } \\
P<0.05\end{array}$ \\
\hline 80 & $7 / F$ & $23.4 \pm 1.4$ & Fit & Treadmill & $2 \times 60 \mathrm{~min}$ & $80 \% \mathrm{Vo}_{2} \max$ & $P<0.05$ \\
\hline
\end{tabular}

(Continued) 
Table I (Continued)

\begin{tabular}{|c|c|c|c|c|c|c|c|}
\hline Publication & N/sex & Age & $\begin{array}{l}\text { Fitness } \\
\text { state }\end{array}$ & $\begin{array}{l}\text { Exercise } \\
\text { type }\end{array}$ & $\begin{array}{l}\text { Duration/ } \\
\text { distance }\end{array}$ & $\begin{array}{l}\text { Intensity } \\
\text { level }\end{array}$ & $\begin{array}{l}\text { Significance } \\
\text { (endorphine } \\
\text { increase) }\end{array}$ \\
\hline 24 & $\begin{array}{l}5 / M+5 / F \\
5 / M+5 / F\end{array}$ & $\begin{array}{l}26.9 \pm 6.7 \\
21.0 \pm 2.9\end{array}$ & $\begin{array}{l}\text { Fit } \\
\text { Fit }\end{array}$ & Cycle ergometer & $3 \times 20 \min$ & $\begin{array}{l}40 \% \mathrm{VO}_{2} \max \\
60 \% \mathrm{VO}_{2} \max \\
80 \% \mathrm{VO}_{2} \max \\
20 \text { min each }\end{array}$ & $\begin{array}{l}\text { NS } \\
\text { NS } \\
P<0.05 \\
P<0.05\end{array}$ \\
\hline 81 & I4/M & $18-25$ & Mixed & Cycle ergometer & $4 \times 30 \mathrm{~min}$ & $75 \% \mathrm{Vo}_{2} \max$ & $P<0.003$ \\
\hline 82 & $10 / F$ & $|8-2|$ & Highly fit & Cycle ergometer & - & $\begin{array}{l}\text { Graded intensity } \\
\text { to exhaustion }\end{array}$ & $P<0.001$ \\
\hline 83 & $8 / M$ & $22.1 \pm 2.7$ & Fit & Cycle ergometer & $90 \mathrm{~min}$ & $65 \%$ watt $\max$ & $P<0.05$ \\
\hline 84 & $\begin{array}{l}11 / M \\
6 / M\end{array}$ & $\begin{array}{l}34 \pm 2.3 \\
38.1 \pm 4.3\end{array}$ & $\begin{array}{l}\text { Highly fit } \\
\text { Fit }\end{array}$ & Ski race & $75.7 \mathrm{~km}$ & Race conditions & $P<0.001$ \\
\hline 85 & $17 / M$ & $39.8 \pm 10.3$ & Mixed & Treadmill & $15 \mathrm{~min}$ & $\begin{array}{l}\text { Graded intensity } \\
\text { to exhaustion }\end{array}$ & $P<0.01$ \\
\hline 86 & $9 / M$ & - & Highly fit & Outdoor running & $\begin{array}{l}70-80 \mathrm{~min} / \\
22 \mathrm{~km}\end{array}$ & Race condition & $P<0.05$ \\
\hline 36 & $17 / M$ & $26(22-32)$ & Fit & Outdoor running & $12 \mathrm{~min}$ & Maximal pace & $P<0.05$ \\
\hline 87 & $\begin{array}{l}4 / \mathrm{M} \\
4 / \mathrm{F}\end{array}$ & $\begin{array}{l}45 \\
34\end{array}$ & Highly fit & Outdoor running & $\begin{array}{l}7 \mathrm{~d} 23 \mathrm{~h} / \\
\mathrm{I}, 000 \mathrm{~km}\end{array}$ & Race condition & NS \\
\hline 88 & $\begin{array}{l}8 / M \\
7 / M \\
7 / M \\
5 / M\end{array}$ & $30.1 \pm 7.2$ & $\begin{array}{l}\text { Highly fit } \\
\text { Highly fit } \\
\text { Highly fit } \\
\text { Highly fit }\end{array}$ & $\begin{array}{l}\text { Outdoor running } \\
\text { Outdoor running } \\
\text { Outdoor running } \\
\text { Disk throwing }\end{array}$ & $\begin{array}{l}10,000 \mathrm{~m} \\
1,500 \mathrm{~m} \\
100 \mathrm{~m}\end{array}$ & Race condition & $\begin{array}{l}P<0.01 \\
P<0.01 \\
P<0.01 \\
\text { NS }\end{array}$ \\
\hline 89 & $\begin{array}{l}10 / M \\
5 / M\end{array}$ & $24-41$ & Highly fit & $\begin{array}{l}\text { Outdoor running } \\
\text { Cycle ergometer }\end{array}$ & $\begin{array}{l}\text { Marathon race } \\
90 \mathrm{~min}\end{array}$ & $\begin{array}{l}\text { Race condition } \\
50 \% \mathrm{VO}_{2} \max \end{array}$ & $\begin{array}{l}P<0.01 \\
P<0.01\end{array}$ \\
\hline 90 & $8 / M$ & $24.6 \pm 2$ & Fit & Cycle ergometer & $89 \pm 1 \mathrm{~min}$ & $65 \%$ watt $\max$ & $P<0.05$ \\
\hline 91 & $8 / F$ & $29.7 \pm 4.0$ & Fit & Aerobic dance & $45 \mathrm{~min}$ & High intensity & Significant \\
\hline 92 & $\begin{array}{l}5 / M \\
5 / F\end{array}$ & $\begin{array}{l}25 \pm 2 \\
24 \pm 2\end{array}$ & Highly fit & $\begin{array}{l}\text { Treadmill } \\
\text { Treadmill } \\
\text { Outdoor running }\end{array}$ & $\begin{array}{l}30 \pm I \mathrm{~min} / \\
29 \pm I \mathrm{~min} \\
57 \pm 6 \mathrm{~s} / 7 \mathrm{I} \pm 8 \\
5-12 \mathrm{~km}\end{array}$ & $\begin{array}{l}\text { Graded intensity } \\
\text { to exhaustion } \\
\text { Anaerobic trial } \\
\text { Aerobic trial }\end{array}$ & $\begin{array}{l}P<0.00 I(\mathrm{M}+\mathrm{F}) \\
P<0.05(\mathrm{M}) \\
P<0.0 \mathrm{I}(\mathrm{F}) \\
P<0.05(\mathrm{M}+\mathrm{F})\end{array}$ \\
\hline 25 & $10 / M$ & $23-36$ & Highly fit & Treadmill & $6 \times 10 \mathrm{~min}$ & $\begin{array}{l}50 / 60 / 70 / 80 \% \\
\mathrm{VO}_{2} \max \\
90 \% \text { and } 100 \% \\
\mathrm{VO}_{2} \max \end{array}$ & $\begin{array}{l}\text { NS } \\
P<0.001\end{array}$ \\
\hline 93 & $23 / M$ & $26.0 \pm 0.9$ & $\begin{array}{l}\text { Fit to } \\
\text { highly fit }\end{array}$ & Rowing ergometer & $9 \min$ & Anaerobic exercise & $P<0.001$ \\
\hline 94 & $8 / M$ & $18-23$ & - & Cycle ergometer & $25 \mathrm{~min}$ & $50 \% \mathrm{VO}_{2} \max$ & $P<0.05$ \\
\hline 95 & $\begin{array}{l}32 / \mathrm{M} \\
9 / \mathrm{F}\end{array}$ & $\begin{array}{l}24-63 \\
25-55\end{array}$ & $\begin{array}{l}\text { Highly fit } \\
\text { Highly fit }\end{array}$ & $\begin{array}{l}\text { Mountain running } \\
\text { Mountain running }\end{array}$ & $\begin{array}{l}46 \mathrm{~km} \\
46 \mathrm{~km}\end{array}$ & $\begin{array}{l}\text {-rRace condition } \\
\text { rRace condition }\end{array}$ & $\begin{array}{l}P<0.001 \\
\text { NS }\end{array}$ \\
\hline 26 & $\begin{array}{l}12 / M \\
I 1 / M\end{array}$ & $\begin{array}{l}23.0 \pm 0.8 \\
21.4 \pm 1.6\end{array}$ & $\begin{array}{l}\text { Fit } \\
\text { Unfit }\end{array}$ & Cycle ergometer & $2 \times 120 \mathrm{~min}$ & $\begin{array}{l}45 \% \mathrm{Vo}_{2} \max \\
60 \% \mathrm{VO}_{2} \max \end{array}$ & $\begin{array}{l}\text { NS } \\
P<0.05 \\
\text { (only fit) }\end{array}$ \\
\hline 27 & $\begin{array}{l}6 / \mathrm{M} \\
6 / \mathrm{F}\end{array}$ & $\begin{array}{l}21.8 \pm 0.7 \\
23.7 \pm 1.4\end{array}$ & - & $\begin{array}{l}\text { Cycle } \\
\text { ergometer }\end{array}$ & $<50$ min & $\begin{array}{l}20 \min 30 \% \\
20 \min 60 \% \\
\text { Ride to exhaustion at } \\
90 \% \mathrm{Vo}_{2} \max \end{array}$ & $\begin{array}{l}\text { NS } \\
P<0.05(F) \\
P<0.01(F+M)\end{array}$ \\
\hline
\end{tabular}

Abbreviations: NS, nonsignificant; HR, heart rate.

to capture endogenous neurotransmitter release in the CNS under experimental challenges (eg, pharmacological, cognitive, or sensorimotor). Currently, studies investigating neurotransmitter release cannot be performed with magnetic resonance imaging or other neuroimaging techniques. The so-called "displacement" or "ligand activation studies" allow quantifying and localizing ligand binding changes by comparing rest and postexercise conditions ${ }^{40}$ Compared with animal work, the major advantage of studying central neurotransmission directly in human athletes is that 
Table 2 Papers reporting peripheral beta-endorphin values in relation to mood changes induced by exercise challenges

\begin{tabular}{|c|c|c|c|}
\hline Publication & Endorphin increase & $\begin{array}{l}\text { Mood } \\
\text { elevation }\end{array}$ & Relationship \\
\hline 13 & $\begin{array}{l}\mathrm{P}<0.05\left(60 \% \mathrm{Vo}_{2} \max \right) \\
\mathrm{NS}\left(80 \% \mathrm{VO}_{2} \max \right)\end{array}$ & NS & No relationship \\
\hline 65 & $P<0.05$ & NS & No relationship \\
\hline 30 & $P<0.000 I$ & Significant & $\begin{array}{l}\text { Positive } \\
\text { relationship }\end{array}$ \\
\hline 67 & $P<0.001$ & NS & No relationship \\
\hline 3 & $P<0.01$ & Significant & $\begin{array}{l}\text { Positive } \\
\text { relationship }\end{array}$ \\
\hline 77 & NS & Significant & No relationship \\
\hline 81 & $P<0.003$ & NS & No relationship \\
\hline
\end{tabular}

Abbreviation: NS, nonsignificant.

detectable binding changes can be tested for correlation with psychophysical effects, as detectable via standardized neuropsychological assessments. Moreover, compared with microdialysis studies in animals, which are restricted to selected brain areas, PET ligand studies provide quantitative measures of tracer binding in the entire human brain.

\section{Basic mechanisms and methodological limitations of PET ligand activation studies}

Ligand activation studies derive quantitative measures of endogenous transmitter trafficking from ligand binding changes that result from competition at specific receptor binding sites in the brain. Although the temporal resolution of PET is low in comparison to other functional brain imaging techniques and does not suffice to capture real-time dynamics of transmitter release in the human brain, PET ligand activation studies allow calculating sustained tracer binding changes induced by previous exercise, ie, manifesting as prolonged changes in ligand binding status. It has to be pointed out that depending on the experimental challenge, either increased or decreased ligand binding changes have been identified in identical brain regions related to fundamentally different experimental challenges, ie, decreased binding reflecting enhanced release of the endogenous transmitter relative to rest,$^{41}$ or increased binding reflecting decreased release of the endogenous transmitter relative to rest. ${ }^{42}$

Table 3 Papers reporting peripheral beta-endorphin values in relation to changes in pain perception induced by exercise challenges

\begin{tabular}{llll}
\hline Publication & $\begin{array}{l}\text { Endorphin } \\
\text { increase }\end{array}$ & $\begin{array}{l}\text { Pain } \\
\text { (hypoalgesia) }\end{array}$ & Relationship \\
\hline 59 & $P<0.0001$ & $\begin{array}{l}P<0.01 \\
\text { Significant }\end{array}$ & $\begin{array}{l}\text { No relationship } \\
\text { Positive } \\
\text { relationship }\end{array}$ \\
31 & $P<0.001$ & Significant & $\begin{array}{l}\text { Positive } \\
\text { relationship }\end{array}$ \\
\hline
\end{tabular}

The methodological details of PET ligand activation studies (eg, study designs, suitable PET tracers, ligand modeling approaches, etc) have been summarized extensively in a recent review article by Boecker et $\mathrm{a}^{40}$ nonetheless, it is important to point out here again that PET is associated with radiation exposure, and, depending on the used tracer, with arterial cannulation for calculating the arterial input function. These methodological issues limit repeated PET acquisitions in the same patient, for instance PET scans under different experimental conditions, or longitudinal study designs. Usually, either separate acquisitions (eg, "baseline" scan and "experimental" scan in counterbalanced order) or one acquisition with an intermediate challenge are performed. In the context of exercise challenge studies, however, intermediate challenges (for instance, using a cycling device installed in the PET unit) are difficult to perform, as exercise challenges risk of being associated with severe head movement artifacts. This will be particularly limiting when intending to study the effects of high intensity or long intensity exercise challenges. Therefore, previous ligand activation work investigating exercise challenges has employed two separated scans, ie, one scan under baseline conditions and one scan immediately after exercise. In such a scenario, PET scanning typically starts with the injection of the radiotracer (to capture the tracer input function) and is continued for a prolonged time period (to capture specific neuroreceptor binding of the PET tracer using parametric and nonparametric kinetic modeling). It has to be considered, however, that enduring experimental challenges, such as continuous exercise, may induce receptor internalization or downregulation, ${ }^{43}$ and can hardly be distinguished by means of PET from decreased binding due to enhanced endogenous transmitter release.

\section{PET ligand activation studies in the sport sciences}

The first study to apply ligand PET to exercising humans was published in 2000 by Wang et al ${ }^{44}$ These authors studied 12 healthy volunteers using the dopaminergic PET tracer ${ }^{11} \mathrm{C}$-Raclopride which binds to striatal D2-receptors. ${ }^{44}$ Examining subjects twice allowed testing for the effects of a 30-minute treadmill exercise (average speed of $8.7 \pm 0.5 \mathrm{~km} / \mathrm{h} ; 5.4 \pm 0.3 \mathrm{mph}$ ) and at an inclination of $3.3^{\circ} \pm 2^{\circ}$ ) upon dopaminergic release; however, no significant differences in binding at the $\mathrm{D} 2$ receptors were identified in this cohort after subjects exercised vigorously for 30 minutes. In the second previous study, which was performed at the Technical University Munich, ${ }^{41} 10$ trained athletes were scanned at rest and after 2 hours of 
outdoor running using the nonselective opioidergic ligand 6-O-(2-[18 $\mathrm{F}]$ fluoroethyl)-6-O-desmethyldiprenorphine $\left(\left[{ }^{18} \mathrm{~F}\right] \mathrm{FDPN}\right)$. These studies were performed to examine whether endogenous opioids are released after exercise and if so, whether such effects are linked to mood changes. In line with the "endorphin hypothesis", this study identified significant reductions in $\left[{ }^{18} \mathrm{~F}\right] \mathrm{FDPN}$ binding in certain regions of the brain. ${ }^{41}$ The strongest effects were seen in orbitofrontal cortex and in areas belonging to the limbic system, including the anterior cingulate cortex and the anterior insula. Although this work has been conducted in a rather small sample of $\mathrm{N}=10$ athletes undergoing 2 PET scans each, it is noteworthy that the displacement effects were clustered in brain regions associated with affective processing and current theories of opioid-mediated pleasure generation ${ }^{45}$ Regression analyses further indicated that the amount of endogenous opioidergic release was inversely correlated to the level of euphoria determined after exercise on visual analog mood scales. ${ }^{41} \mathrm{We}$, therefore, conclude that ligand PET is able to noninvasively monitor transmitter release induced by exercise and to link these central neurotransmitter effects to behavioral measures that will inform researchers about the biology of exercise.

Based on this initial feasibility study, future PET work should try to image larger samples of athletes, possibly using subtype-selective tracers like ${ }^{11} \mathrm{C}$-Carfentanil that do not require arterial cannulation. Furthermore, future work should attempt to stratify individual exercise challenges based on appropriate physical fitness tests. Moreover, careful monitoring of exercise load, use of appropriate neuropsychological tests designed for repeated testing, and incorporation of biomarkers like lactate, beta-endorphins, etc would advance PET ligand applications in the field of exercise research. Considering the duration of PET ligand studies, an improvement to our previous work would be to avoid any time delay between the end of exercise and the beginning of the study (injection of the PET tracer). This might be achieved with tracers that do not require arterial cannulation and may, thereby, be more suitable to detect the full range of neurotransmitter change in the immediate postexercise period. Of course, it is well conceivable that the extent of detectable neurotransmitter change may be further amplified with more strenuous exercise challenges, but these various effects will have to be systematically studied in the future. Having said this, the investigation of other neurotransmitter systems such as the endocannabinoid system ${ }^{46,47}$ or the serotonergic system may represent alternative targets for future studies.

\section{Conclusion}

The value of measuring plasma levels of neurotransmitters for understanding the neuro-psychophysiology of exercise is uncertain. Based on a literature survey spanning approximately 3 decades, we conclude that beta-endorphins levels in plasma after exercise challenges gives only limited information regarding putative central opioidergic effects and, thus, functional mechanisms of behavioral change. In particular, the amount of neurotransmitter detectable in the peripheral blood does not mirror the magnitude of central neurotransmission.

PET is proposed here as a powerful tool for human exercise research, with a hitherto unprecedented potential to unravel the "missing link" between neurotransmission in the CNS under exercise conditions and associated neurobehavioral effects in athletes. It is expected that PET will allow advancing our understanding of underlying neurochemical mechanisms involved in physical exercise. In particular, the correlation between neurochemistry and validated neuropsychological assessments has a strong potential to advance our knowledge about the central underpinnings of exerciseinduced psychophysical effects. In conclusion, although still in its infancy, PET ligand activation studies provide a powerful tool for human exercise research.

\section{Disclosure}

The authors report no conflicts of interest in this work. Henning Boecker holds an endowed professorship (Philips).

\section{References}

1. Morgan WP. Affective beneficence of vigorous physical activity. Med Sci Sports Exerc. 1985;17:94-100.

2. Rosch PJ. Exercise and stress reduction. Compr Ther. 1985;11:10-15.

3. Janal MN, Colt EW, Clark WC, Glusman M. Pain sensitivity, mood and plasma endocrine levels in man following long-distance running: effects of naloxone. Pain. 1984;19:13-25.

4. Wildmann J, Kruger A, Schmole M, et al. Increase of circulating beta-endorphin-like immunoreactivity correlates with the change in feeling of pleasantness after running. Life Sci. 1986;38: 997-1003.

5. Pierce EF, Pate DW. Mood alterations in older adults following acute exercise. Percept Mot Skills. 1994;79:191-194.

6. Meeusen R, Piacentini MF, de Meirleir K. Brain microdialysis in exercise research. Sports Med. 2001;31:965-983.

7. de Souza CG, Michelini LC, Fior-Chadi DR. Receptor changes in the nucleus tractus solitarii of the rat after exercise training. Med Sci Sports Exerc. 2001;33:1471-1476.

8. Chaouloff F. Physical exercise and brain monoamines: a review. Acta Physiol Scand. 1989;137:1-13.

9. Sforzo GA. Opioids and exercise. An update. Sports Med. 1989;7: 109-124.

10. Harber VJ, Sutton JR. Endorphins and exercise. Sports Med. 1984;1:154-171.

11. Francis K. The role of endorphins in exercise: a review of current knowledge. J Orthop Sports Phys Ther. 1983;4:169-173.

12. Sheps DS, Koch G, Bragdon EE, et al. The reproducibility of resting and post exercise plasma beta-endorphins. Life Sci. 1988;43:787-791. 
13. Farrell PA, Gates WK, Maksud MG, Morgan WP. Increases in plasma beta-endorphin/beta-lipotropin immunoreactivity after treadmill running in humans. J Appl Physiol. 1982;52:1245-1249.

14. Goldfarb AH, Jamurtas AZ. Beta-endorphin response to exercise. An update. Sports Med. 1997;24:8-16.

15. Kraemer WJ, Ratamess NA. Hormonal responses and adaptations to resistance exercise and training. Sports Med. 2005;35:339-361.

16. Bullen BA, Skrinar GS, Beitins IZ, et al. Endurance training effects on plasma hormonal responsiveness and sex hormone excretion. $J$ Appl Physiol. 1984;56:1453-1463.

17. de Vries WR, Bernards NT, de Rooij MH, Koppeschaar HP. Dynamic exercise discloses different time-related responses in stress hormones. Psychosom Med. 2000;62:866-872.

18. Farrell PA, Kjaer M, Bach FW, Galbo H. Beta-endorphin and adrenocorticotropin response to supramaximal treadmill exercise in trained and untrained males. Acta Physiol Scand. 1987;130: 619-625.

19. Goldfarb AH, Hatfield BD, Armstrong D, Potts J. Plasma betaendorphin concentration: response to intensity and duration of exercise. Med Sci Sports Exerc. 1990;22:241-244.

20. Goldfarb AH, Hatfield BD, Potts J, Armstrong D. Beta-endorphin time course response to intensity of exercise: effect of training status. Int $J$ Sports Med. 1991;12:264-268.

21. Goldfarb AH, Jamurtas AZ, Kamimori GH, et al. Gender effect on beta-endorphin response to exercise. Med Sci Sports Exerc. 1998;30: 1672-1676.

22. Laurent D, Schneider KE, Prusaczyk WK, et al. Effects of caffeine on muscle glycogen utilization and the neuroendocrine axis during exercise. J Clin Endocrinol Metab. 2000;85:2170-2175.

23. McMurray RG, Forsythe WA, Mar MH, Hardy CJ. Exercise intensityrelated responses of beta-endorphin and catecholamines. Med Sci Sports Exerc. 1987;19:570-574.

24. McMurray RG, Hardy CJ, Roberts S, et al. Neuroendocrine responses of type A individuals to exercise. Behav Med. 1989;15:84-92.

25. Rahkila P, Hakala E, Alen M, et al. Beta-endorphin and corticotropin release is dependent on a threshold intensity of running exercise in male endurance athletes. Life Sci. 1988;43:551-558.

26. Viru A, Tendzegolskis Z. Plasma endorphin species during dynamic exercise in humans. Clin Physiol. 1995;15:73-79.

27. Viswanathan M, van Dijk JP, Graham TE, et al. Exercise- and coldinduced changes in plasma beta-endorphin and beta-lipotropin in men and women. J Appl Physiol. 1987;62:622-627.

28. Dishman RK, O'Connor PJ. Lessons in exercise neurobiology: the case of endorphins. Ment Health Phys Act. 2009;2:4-9.

29. Dearman J, Francis KT. Plasma levels of catecholamines, cortisol, and beta-endorphins in male athletes after running 26.2, 6, and 2 miles. J Sports Med Phys Fitness. 1983;23:30-38.

30. Harte JL, Eifert GH, Smith R. The effects of running and meditation on beta-endorphin, corticotropin-releasing hormone and cortisol in plasma, and on mood. Biol Psychol. 1995;40:251-265.

31. Hartmann S, Bung P, Schlebusch H, Hollmann W. The analgesic effect of exercise during labor. Z Geburtshilfe Neonatol. 2005;209: 144-150.

32. Droste C. Physical exercise, endogenous opiates and pain regulation. Schmerz. 1991;5:138-147.

33. Edwards L, Ring C, France CR, et al. Effects of opioid blockade on nociceptive flexion reflex thresholds and nociceptive responding in hypertensive and normotensive individuals. Int J Psychophysiol. 2008;69:96-100.

34. Gordon NF, Duncan JJ, Kohl HW. Effect of opioid antagonism on the ability to tolerate maximal anaerobic exercise. S Afr Med J. 1989;76: 268-269.

35. Ray CA, Carter JR. Central modulation of exercise-induced muscle pain in humans. J Physiol. 2007;585:287-294.

36. Paulev PE, Thorboll JE, Nielsen U, et al. Opioid involvement in the perception of pain due to endurance exercise in trained man. Jpn J Physiol. 1989;39:67-74.
37. Jarvekulg A, Viru A. Opioid receptor blockade eliminates mood effects of aerobic gymnastics. Int J Sports Med. 2002;23:155-157.

38. Daniel M, Martin AD, Carter J. Opiate receptor blockade by naltrexone and mood state after acute physical activity. Br J Sports Med. 1992; 26:111-115.

39. Radosevich PM, Nash JA, Lacy DB, et al. Effects of low- and highintensity exercise on plasma and cerebrospinal fluid levels of ir-betaendorphin, ACTH, cortisol, norepinephrine and glucose in the conscious dog. Brain Res. 1989;498:89-98.

40. Boecker H, Henriksen G, Sprenger T, et al. Positron emission tomography ligand activation studies in the sports sciences: measuring neurochemistry in vivo. Methods. 2008;45:307-318.

41. Boecker H, Sprenger T, Spilker ME, et al. The runner's high: opioidergic mechanisms in the human brain. Cereb Cortex. 2008;18:2523-2531.

42. Zubieta JK, Ketter TA, Bueller JA, et al. Regulation of human affective responses by anterior cingulate and limbic mu-opioid neurotransmission. Arch Gen Psychiatry. 2003;60:1145-1153.

43. Laruelle M. Imaging synaptic neurotransmission with in vivo binding competition techniques: a critical review. J Cereb Blood Flow Metab. 2000;20:423-451

44. Wang GJ, Volkow ND, Fowler JS, et al. PET studies of the effects of aerobic exercise on human striatal dopamine release. $J$ Nucl $\mathrm{Med}$. 2000;41:1352-1356.

45. Kringelbach ML, Berridge KC. Towards a functional neuroanatomy of pleasure and happiness. Trends Cogn Sci. 2009;13:479-487.

46. Dietrich A, McDaniel WF. Endocannabinoids and exercise. Br J Sports Med. 2004;38:536-541.

47. Sparling PB, Giuffrida A, Piomelli D, et al. Exercise activates the endocannabinoid system. Neuroreport. 2003;14:2209-2211.

48. Angelopoulos TJ. Beta-endorphin immunoreactivity during highintensity exercise with and without opiate blockade. Eur J Appl Physiol. 2001;86:92-96.

49. Armstrong DW 3rd, Hatfield BD. Hormonal responses to opioid receptor blockade: during rest and exercise in cold and hot environments. Eur J Appl Physiol. 2006;97:43-51.

50. Brooks S, Burrin J, Cheetham ME, et al. The responses of the catecholamines and beta-endorphin to brief maximal exercise in man. Eur J Appl Physiol Occup Physiol. 1988;57:230-234.

51. Bouix O, Brun JF, Fedou C, et al. Plasma beta-endorphin, corticotrophin and growth hormone responses to exercise in pubertal and prepubertal children. Horm Metab Res. 1994;26:195-199.

52. Carrasco L, Villaverde C, Oltras CM. Endorphin responses to stress induced by competitive swimming event. J Sports Med Phys Fitness. 2007;47:239-245

53. Colt EW, Wardlaw SL, Frantz AG. The effect of running on plasma beta-endorphin. Life Sci. 1981;28:1637-1640.

54. de Diego Acosta AM, Garcia JC, Fernandez-Pastor VJ, et al. Influence of fitness on the integrated neuroendocrine response to aerobic exercise until exhaustion. J Physiol Biochem. 2001;57:313-320.

55. de Meirleir K, Naaktgeboren N, van Steirteghem A, Block P. Plasma beta-endorphin levels after exercise. J Endocrinol Invest. 1985;8:89.

56. de Meirleir K, Naaktgeboren N, van Steirteghem A, et al. Betaendorphin and ACTH levels in peripheral blood during and after aerobic and anaerobic exercise. Eur J Appl Physiol Occup Physiol. 1986;55:5-8.

57. Di Luigi L, Guidetti L, Baldari C, Romanelli F. Heredity and pituitary response to exercise-related stress in trained men. Int J Sports Med. 2003;24:551-558.

58. Donevan RH, Andrew GM. Plasma beta-endorphin immunoreactivity during graded cycle ergometry. Med Sci Sports Exerc. 1987;19: 229-233.

59. Droste C, Greenlee MW, Schreck M, Roskamm H. Experimental pain thresholds and plasma beta-endorphin levels during exercise. Med Sci Sports Exerc. 1991;23:334-342.

60. Elias AN, Fairshter R, Pandian MR, et al. Beta-endorphin/beta-lipotropin release and gonadotropin secretion after acute exercise in physically conditioned males. Eur J Appl Physiol Occup Physiol. 1989;58: $522-527$. 
61. Elliot DL, Goldberg L, Watts WJ, Orwoll E. Resistance exercise and plasma beta-endorphin/beta-lipotrophin immunoreactivity. Life Sci. 1984;34:515-518.

62. Gannon GA, Rhind SG, Suzui M, et al. Beta-endorphin and natural killer cell cytolytic activity during prolonged exercise. Is there a connection? Am J Physiol. 1998;275:R1725-R1734.

63. Gerra G, Volpi R, Delsignore R, et al. ACTH and beta-endorphin responses to physical exercise in adolescent women tested for anxiety and frustration. Psychiatry Res. 1992;41:179-186.

64. Glamsta EL, Morkrid L, Lantz I, Nyberg F. Concomitant increase in blood plasma levels of immunoreactive hemorphin-7 and betaendorphin following long distance running. Regul Pept. 1993;49:9-18.

65. Goldfarb AH, Hatfield BD, Sforzo GA, Flynn MG. Serum betaendorphin levels during a graded exercise test to exhaustion. $\mathrm{Med} \mathrm{Sci}$ Sports Exerc. 1987;19:78-82.

66. Harbach H, Hell K, Gramsch C, et al. Beta-endorphin (1-31) in the plasma of male volunteers undergoing physical exercise. Psychoneuroendocrinology. 2000;25:551-562.

67. Hatfield BD, Goldfarb AH, Sforzo GA, Flynn MG. Serum betaendorphin and affective responses to graded exercise in young and elderly men. J Gerontol. 1987;42:429-431.

68. Heitkamp HC, Schmid K, Scheib K. Beta-endorphin and adrenocorticotropic hormone production during marathon and incremental exercise. Eur J Appl Physiol Occup Physiol. 1993;66:269-274.

69. Heitkamp HC, Huber W, Scheib K. Beta-endorphin and adrenocorticotrophin after incremental exercise and marathon running - female responses. Eur J Appl Physiol Occup Physiol. 1996;72:417-424.

70. Heitkamp HC, Schulz H, Rocker K, Dickhuth HH. Endurance training in females: changes in beta-endorphin and ACTH. Int J Sports Med. 1998;19:260-264

71. Hickey MS, Franke WD, Herbert WG, et al. Opioid antagonism, perceived exertion and tolerance to exercise-thermal stress. Int J Sports Med. 1992;13:326-331.

72. Howlett TA, Tomlin S, Ngahfoong L, et al. Release of beta endorphin and met-enkephalin during exercise in normal women: response to training. Br Med J (Clin Res Ed). 1984;288:1950-1952.

73. Kelso TB, HerbertWG, Gwazdauskas FC, et al. Exercise-thermoregulatory stress and increased plasma beta-endorphin/beta-lipotropin in humans. J Appl Physiol. 1984;57:444-449.

74. Kraemer WJ, Patton JF, Knuttgen HG, et al. Hypothalamic-pituitaryadrenal responses to short-duration high-intensity cycle exercise. $J \mathrm{Appl}$ Physiol. 1989;66:161-166.

75. Kraemer WJ, Fleck SJ, Callister R, et al. Training responses of plasma beta-endorphin, adrenocorticotropin, and cortisol. Med Sci Sports Exerc. 1989;21:146-153.

76. Kraemer WJ, Hamilton AJ, Gordon SE, et al. Plasma changes in betaendorphin to acute hypobaric hypoxia and high intensity exercise. Aviat Space Environ Med. 1991;62:754-758.

77. Kraemer RR, Dzewaltowski DA, Blair MS, et al. Mood alteration from treadmill running and its relationship to beta-endorphin, corticotropin, and growth hormone. J Sports Med Phys Fitness. 1990;30:241-246.

78. Langenfeld ME, Hart LS, Kao PC. Plasma beta-endorphin responses to one-hour bicycling and running at $60 \% \mathrm{VO}_{2} \max$. Med Sci Sports Exerc. 1987;19:83-86.
79. Louisy F, Guezennec CY, Lartigue M, et al. Influence of endogenous opioids on atrial natriuretic factor release during exercise in man. Eur J Appl Physiol Occup Physiol. 1989;59:34-38.

80. McMurray RG, Fafrowicz JF, Berry M. The endorphin response of women to sleep loss and exercise. Aviat Space Environ Med. 1988; 59:129-132.

81. McMurray RG, Hill D, Field KM. Diurnal variations of beta-endorphin at rest and after moderate intensity exercise. Chronobiol Int. 1990; 7:135-142.

82. Mesaki N, Sasaki J, Motobu M, et al. Effect of naloxone on hormonal changes during exercise. Nippon Sanka Fujinka Gakkai Zasshi. 1989;41:1991-1998.

83. Meeusen R, Piacentini MF, van Den Eynde S, et al. Exercise performance is not influenced by a 5-HT reuptake inhibitor. Int J Sports Med. 2001;22:329-336

84. Mougin C, Henriet MT, Baulay A, et al. Plasma levels of beta-endorphin, prolactin and gonadotropins in male athletes after an international nordic ski race. Eur J Appl Physiol Occup Physiol. 1988;57:425-429.

85. Oleshansky MA, Zoltick JM, Herman RH, et al. The influence of fitness on neuroendocrine responses to exhaustive treadmill exercise. Eur $J$ Appl Physiol Occup Physiol. 1990;59:405-410.

86. Oltras CM, Mora F, Vives F. Beta-endorphin and ACTH in plasma: effects of physical and psychological stress. Life Sci. 1987;40:1683-1686.

87. Pestell RG, Hurley DM, Vandongen R. Biochemical and hormonal changes during a $1000 \mathrm{~km}$ ultramarathon. Clin Exp Pharmacol Physiol. 1989;16:353-361.

88. Petraglia F, Barletta C, Facchinetti F, et al. Response of circulating adrenocorticotropin, beta-endorphin, beta-lipotropin and cortisol to athletic competition. Acta Endocrinol (Copenh). 1988;118:332-336.

89. Petraglia F, Bacchi Modena A, Comitini G, et al. Plasma betaendorphin and beta-lipotropin levels increase in well trained athletes after competition and non competitive exercise. J Endocrinol Invest. 1990;13:19-23.

90. Piacentini MF, Meeusen R, Buyse L, et al. Hormonal responses during prolonged exercise are influenced by a selective DA/NA reuptake inhibitor. Br J Sports Med. 2004;38:129-133.

91. Pierce EF, Eastman NW, Tripathi HL, et al. Beta-endorphin response to endurance exercise: relationship to exercise dependence. Percept Mot Skills. 1993;77:767-770.

92. Rahkila P, Hakala E, Salminen K, Laatikainen T. Response of plasma endorphins to running exercises in male and female endurance athletes. Med Sci Sports Exerc. 1987;19:451-455.

93. Schulz A, Harbach H, Katz N, et al. Beta-endorphin immunoreactive material and authentic beta-endorphin in the plasma of males undergoing anaerobic exercise on a rowing ergometer. Int J Sports Med. 2000;21:513-517.

94. Shen A, Chin J, Fullerton M, et al. Increases in plasma beta-endorphin concentrations during exercise do not contribute to increases in heart rate following autonomic blockade in man. Br J Clin Pharmacol. 1992;33:89-92.

95. Strassman RJ, Appenzeller O, Lewy AJ, et al. Increase in plasma melatonin, beta-endorphin, and cortisol after a 28.5-mile mountain race: relationship to performance and lack of effect of naltrexone. $J$ Clin Endocrinol Metab. 1989;69:540-545.

Open Access Journal of Sports Medicine

\section{Publish your work in this journal}

Open Access Journal of Sports Medicine is an international, peer-reviewed, open access journal publishing original research, reports, reviews and commentaries on all areas of sports medicine. The manuscript management system is completely online and includes a very quick and fair peer-review system. 